\title{
Dual-source dual-energy thin-section CT combined with small field of view technique for small lymph node in thyroid cancer: a retrospective diagnostic study
}

\author{
Shuiqing Zhuo ${ }^{1 \#}$, Jiayuan Sun ${ }^{2 \#}$, Jinyong Chang ${ }^{3}$, Longzhong Liu ${ }^{4}$, Sheng $\mathrm{Li}^{1}$ \\ ${ }^{1}$ Department of Radiology, Sun Yat-sen University Cancer Center, State Key Laboratory of Oncology in South China, Collaborative Innovation \\ Center for Cancer Medicine, Guangzhou, China; ${ }^{2}$ Department of Radiation Oncology, Sun Yat-sen University Cancer Center, State Key Laboratory \\ of Oncology in South China, Collaborative Innovation Center for Cancer Medicine, Guangzhou, China; ${ }^{3}$ Department of Radiology, Lian Jiang \\ People's Hospital, Lianjiang, China; ${ }^{4}$ Department of Ultrasound, Sun Yat-sen University Cancer Center, State Key Laboratory of Oncology in South \\ China, Collaborative Innovation Center for Cancer Medicine, Guangzhou, China \\ Contributions: (I) Conception and design: S Li; (II) Administrative support: S Zhuo, S Li; (III) Provision of study materials or patients: S Zhuo, S Li, \\ L Liu; (IV) Collection and assembly of data: S Zhuo, J Sun, J Chang, L Liu; (V) Data analysis and interpretation: S Li, J Chang; (VI) Manuscript \\ writing: All authors; (VII) Final approval of manuscript: All authors. \\ \#These authors contributed equally to this work. \\ Correspondence to: Sheng Li. Department of Radiology, Sun Yat-sen University Cancer Center, 651 Dongfeng Road East, Guangzhou, Guangdong \\ 510060, China. Email: lisheng@sysucc.org.cn; Longzhong Liu. Department of Ultrasound, Sun Yat-sen University Cancer Center, 651 Dongfeng \\ Road East, Guangzhou, Guangdong 510060, China. Email: liulzh@sysucc.org.cn.
}

Background: To evaluate the diagnostic performance of quantitative spectral parameters derived from dual-source dual-energy CT at small field of view (FOV) for small lymph node metastasis in thyroid cancer.

Methods: This was a retrospective diagnostic study. From 2016 to 2019, 280 patients with thyroid disease underwent thin-section dual-source dual-energy thyroid CT and thyroid surgery. The data of patients with lymph nodes having a short diameter of 2-6 $\mathrm{mm}$ was analyzed. The quantitative dual-energy CT parameters of targeted lymph nodes were measured, and all parameters between metastatic and nonmetastatic lymph nodes were compared. These parameters were then fitted to univariable and multivariable binary logistic regression models. The diagnostic role of spectral parameters was analyzed by receiver operating characteristic (ROC) curves and compared with the McNemar test. Small FOV CT images and a mathematical model were used to judge the status of lymph nodes respectively, and then compared with the golden criterion-pathological diagnosis. The cut-off value of the model was 0.4419 , with a sensitivity of $90.2 \%$ and a specificity of $92.7 \%$.

Results: Of the 216 lymph nodes investigated in this study, $52.3 \%$ and $23.6 \%$ had a short diameter of 2-3 and $4 \mathrm{~mm}$, respectively. Multiple quantitative CT parameters were significantly different between benign and malignant lymph nodes, and binary regression analysis was performed. The mathematical model was: $\mathrm{p}=\mathrm{ey} /(1+\mathrm{ey}), \mathrm{y}=-23.119+0.033 \times$ precontrast electron cloud density $+0.076 \times$ arterial phase normalized iodine concentration $+2.156 \times$ arterial phase normalized effective atomic number $-0.540 \times$ venous phase slope of the spectral Hounsfield unit curve $+1.676 \times$ venous phase iodine concentration. This parameter model had an AUC of $92 \%$, with good discrimination and consistency, and the diagnostic accuracy was $90.3 \%$. The diagnostic accuracy of the CT image model was $43.1 \%$, and for lymph nodes with a short-diameter of 2-3 mm, the diagnostic accuracy was $22.1 \%$.

Conclusions: The parameter model showed higher diagnostic accuracy than the CT image model for diagnosing small lymph node metastasis in thyroid cancer, and quantitative dual-energy CT parameters were very useful for small lymph nodes that were difficult to be diagnosed only on conventional CT images.

Trial registration: This study is retrospectively registered, and we have registered a prospective study (Registration number: ChiCTR2000035195; http://www.chictr.org.cn). 


\begin{abstract}
Keywords: Dual-source dual-energy CT; small field of view (small FOV); thyroid cancer; lymph node; radiologicpathological correlation
\end{abstract}

Submitted Nov 11, 2020. Accepted for publication Feb 23, 2021.

doi: $10.21037 /$ gs-20-822

View this article at: http://dx.doi.org/10.21037/gs-20-822

\section{Introduction}

Thyroid cancer is one of the most common malignant tumors with increasing prevalence in recent years. Lymph node metastasis is also widespread, and its extent determines surgical planning. Based on the American Thyroid Association guidelines, ultrasound is the recommended imaging modality to evaluate cervical lymph node status, while computed tomography (CT) is suggested as an adjunct (1). However, ultrasound has limitations for detecting LN metastasis in tricky areas due to gas, bone, or deep locations. Further, since macrometastasis in lymph nodes originally develops from micrometastasis and all large metastatic lymph nodes develop from small metastatic lymph nodes, therefore, micrometastatic LNs or metastasis in small LNs can be considered as the early stage of lymph node metastasis (2). In order to find out lymph node metastasis at an early stage, it is necessary to investigate small lymph node metastasis. However, the detection and diagnosis of very small lymph nodes or micrometastatic lymph nodes are quite cumbersome using CT or ultrasound. Small lymph nodes are difficult to be diagnosed only on conventional CT images because there are few metastatic features on CT images. When conventional CT images cannot be used to diagnose some diseases, dual-source dual-energy CT with multiple parameters can be useful, by using $\lambda_{\mathrm{HU}}$, iodine concentration, dual energy index and effective atomic number to identify different lesions. For example, even for malignant and benign lesions with the same CT value, effective atomic number can be used to differentiate them.

Recently, dual-source dual-energy thyroid CT has shown a promising ability for detecting cervical lymph node status of thyroid cancer $(3,4)$. Its CT images at small field of view (FOV) with improved spatial and contrast resolution has a smaller range of scattering artifacts and can detect LN boundary more sharply, which can be visualized in geometrically enlarged images, highlighting the details of small LNs that are often difficult to be shown on CT images at normal FOV. Further, thin-section CT images at small FOV is additionally useful for accurately delineating and diagnosing the LNs. Some dual-energy parameters such as dual-energy index are seldomly reported, and are they helpful in diagnosing lymph node status? Will the above techniques help diagnose small lymph nodes that were difficult to diagnose only on conventional CT images?

Till present, there has been no study investigating the diagnostic accuracy of dual-energy CT for small LN metastasis in patients with thyroid cancer. In this study, we aimed to examine the diagnostic performance of thinsection dual-source dual-energy thyroid CT at small FOV for detecting small cervical LNs in thyroid cancer and study its radiologic-pathological correlation.

We present the following article in accordance with the STARD reporting checklist (available at http://dx.doi. org/10.21037/gs-20-822).

\section{Methods}

\section{Patients}

This was a single-center retrospective diagnostic study, approved by the institutional ethics committee of Sun Yat-sen University Cancer Center (IRB number: B2020156), and informed consent was waived. The study was conducted in accordance with the Declaration of Helsinki (as revised in 2013). From December 2016 to October 2019, the data of 280 patients who underwent thin-section dualsource dual-energy thyroid CT and thyroid surgery with lymphadenectomy at Sun Yat-sen University Cancer Center were retrieved. A total of 216 lymph nodes in 74 patients were enrolled. The enrollment process is illustrated in Figure 1.

Cases were selected based on the following inclusion criteria: (I) the maximal short diameter of all selected cervical lymph nodes should be within the range of 2-6 $\mathrm{mm}$. (II) The pathological results of all lymph nodes at the same level should be the same, and their status was confirmed by pathologic examination. (III) The lymph nodes from level VI were further divided into four sublevels during surgery, including the prelaryngeal, the pretracheal, the 


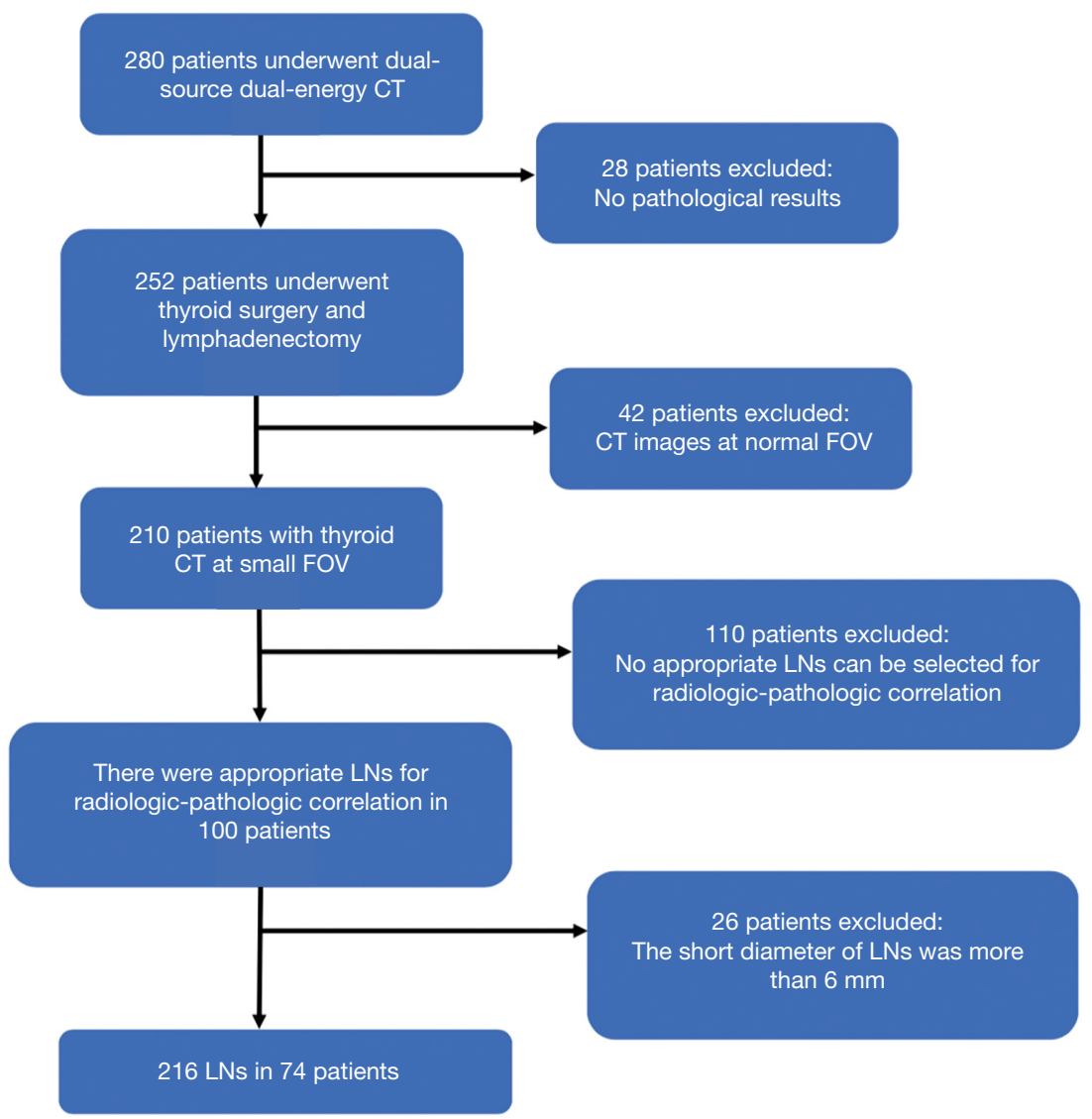

Figure 1 Patient recruitment workflow. In total, 216 out of 74 patients were included according to the selection criteria. The included patients were examined by dual-energy thin-section thyroid CT at 140/160 mm FOV, and had complete clinical and pathological information needed for the study. CT, computed tomography.

right and the left paratracheal nodes. Lymph nodes from each sublevel were all metastatic or normal lymph nodes, so as to facilitate lymph node selection and radiologicpathological correlation. (IV) Preoperative ultrasound showed no suspicious cervical lymph nodes in level $\mathrm{Vb}$. A small number of lymph nodes were located at specific anatomical locations, which were easy to match and locate on CT images and pathological slices, and they were also included in this study.

All the LNs were labeled after resection according to their cervical compartments, and those from cervical level VI were further divided into 4 sublevels. Patients in our study were with thyroid cancer or nodular goiter, and some were also with Hashimoto's thyroiditis.

\section{Quantitative parameters of dual-source dual-energy thin- section CT}

Dual-energy scan mode was used with Siemens dual-source CT (SOMATOM Force CT, SIEMENS, German), and the scan range was from the level of the skull base to the upper edge of the aortic arch. All patients underwent thinsection dual-energy thyroid CT in the precontrast phase, arterial phase, and venous phase. Parameters of dual-energy scanning mode were as follows: two tubes (A and B) were used, with tube voltage /tube current $90 \mathrm{kV} / 250 \mathrm{~mA}$ and Sn 150 kV/125 mA, respectively. Turn on CARE Dose $4 \mathrm{D}$, and the collimation was $2 \mathrm{~mm} \times 192 \mathrm{~mm} \times 0.6 \mathrm{~mm}$. CT images were obtained with a gantry rotation of $0.5 \mathrm{~s}$ 
and pitch factor $1 . \mathrm{A}, \mathrm{B}$ and $\mathrm{M}$ groups of CT images were automatically obtained after scanning. Images of group $\mathrm{A}$ and $\mathrm{B}$ were obtained from low and high tube voltages, respectively. $M$ group of images were mixed energy images for the diagnosis, from linear fusion, with dualenergy fusion coefficient 0.6. The CT images with the slice thickness of $0.75 \mathrm{~mm}$ and layer spacing of 0 were reconstructed at a $512 \times 512$ matrix size. CT images at small FOV was performed in the precontrast, arterial and venous phase. According to neck size, the FOV sizes were $140 \mathrm{~mm}$ $\times 140 \mathrm{~mm}$ or $160 \mathrm{~mm} \times 160 \mathrm{~mm}$. The patients were all in the supine position during the examination. High-pressure syringe was used. The contrast agent used was iopromide $(370 \mathrm{mgI} / \mathrm{mL}$ ) and was injected through the elbow vein at a flow rate of $4.0 \mathrm{~mL} / \mathrm{s}$. The arterial phase scan was automatically triggered. The trigger point was the aortic arch, and the trigger threshold was $100 \mathrm{HU}$. The venous phase was then delayed by $50 \mathrm{~s}$. All images were transferred to the PACS terminal and workstation. The basic signs of lesion morphology evaluation (conventional CT images) and multi-parameter analysis of energy spectrum were performed using the software Syngo.via. Multi-planar reconstructions were performed for axial, coronal, and sagittal planes, with a slice thickness of $0.75 \mathrm{~mm}$. The dual-source dual-energy CT data sets were reconstructed into monochromatic image sets, iodine-based materialdecomposition images, and effective atomic number images.

The region of interest (ROI) was drawn to be as large as possible to include the entire lymph node to obtain the slope of the spectral Hounsfield unit curve. Internal fat, necrosis, and calcifications were not excluded. Further, attention to avoid the peripheral fat tissues. To gain electron cloud density (Rho) and effective atomic number ( $\mathrm{Z}$ ), we drew the ROI according to the boundary of the selected LN. That is, the entire lymph node was included. Small LNs were conformally sketched to accurately outline their contour, and we could sketch lymph nodes with a short diameter of 2-3 mm. All measurements were performed twice on 2 consecutive maximal slices in the selected lymph nodes, and their average values were calculated.

Dual-source dual-energy thyroid CT was used for 280 patients with gemstone spectral imaging (GSI) mode. GSI quantitative parameters, including iodine concentration (IC), normalized iodine concentrations (NIC), slope of the spectral Hounsfield unit curve $\left(\lambda_{\mathrm{HU}}\right)$, effective atomic number, electron cloud density, and double energy index (DEI) in the precontrast, arterial and venous phase, were measured. The iodine concentration of the LNs was respectively divided by the iodine concentration of the aorta to obtain the normalized iodine concentration. The slope of the spectral Hounsfield unit curve, which was defined as the difference between the CT value at $40 \mathrm{keV}$ and that at $70 \mathrm{keV}$ divided by the energy difference $(30 \mathrm{keV})$, was calculated as using the following formula: $\lambda_{\mathrm{HU}}=\left(\mathrm{HU}_{40 \mathrm{keV}}{ }^{-}\right.$ $\left.2013 \mathrm{HU}_{70 \mathrm{keV}}\right) / 30 \mathrm{keV}$, where $\mathrm{HU}_{40 \mathrm{keV}}$ represents the CT value measured on $40-\mathrm{keV}$ images and $\mathrm{HU}_{70 \mathrm{keV}}$ stands for the CT value measured on $70-\mathrm{keV}$ images. CT values of the target $\mathrm{LN}$ in the precontrast, arterial and venous phase were measured on $40-\mathrm{keV}$ images and $70-\mathrm{keV}$ images, respectively. Iodine concentration of the $\mathrm{LN}$ and the aorta were measured on iodine-based material-decomposition images.

\section{Lymph node selection}

In order to ensure that the lymph nodes selected on CT images corresponded to the pathological results, we have adopted the following methods. (I) Some benign lymph nodes originated from the cervical lymph nodes of benign thyroid disease, such as nodular goiter or chronic lymphocytic thyroiditis, and these 20 patients had no malignant tumor. (II) Other benign lymph nodes, which were from patients with thyroid cancer, were confirmed by the pathological results. For example, the pathological results reported that all level IV lymph nodes at the right neck were benign, and some of these LNs were selected as target ones to measure quantitative parameters. The cervical lymph nodes in some patients with thyroid cancer were all non-metastatic lymph nodes, confirmed by postoperative pathological results. We selected some of these lymph nodes for radiologic-pathological comparison. (III) Metastatic lymph nodes were from cervical lymph nodes in patients with thyroid cancer, and all lymph nodes in one level or compartment were reported as metastasis postoperatively. For example, the pathological report showed that all pretracheal nodes were metastatic, and then we selected some of them for CT-pathological comparison. (IV) Some small lymph nodes were located at specific anatomical sites and their locations were labeled in pathological reports. These lymph nodes were also included in our study.

We further divided level VI into four sublevels for more lymph nodes to assess their radiologic-pathological correlation; i.e., all lymph nodes in this sublevel were metastatic lymph nodes or non-metastatic nodes. If the entire LNs were benign or malignant according to postoperative pathological results, these LNs were selected 
as target ones for measuring spectral CT parameters.

For lymph nodes selected for multi-parameter measurement, two radiologists (10 years' experience in thyroid imaging) used plain and dual phases enhanced images to diagnose whether these lymph nodes were metastatic or not, without prior knowledge of the patients' pathological results. Any disagreement was achieved by joint reviews of the CT images. Then, dual-energy CT parameters were used to diagnose these LNs again. At last, we compared the diagnostic performance of the two models: the image model was based on thin-section CT images at small FOV, and the parameter model was based on multiple dual-energy CT parameters.

In our study, the definition of diagnostic difficulty for LNs on thin-section CT images at 140 or $160 \mathrm{~mm}$ FOV was as follows. Some LNs were usually too small (with a short diameter of 2-3 $\mathrm{mm}$ ), without significant signs of metastasis, and they were usually located at level VI and on the same side as the thyroid cancer. In this situation, it was difficult for radiologists to judge if one lymph node was malignant or benign on CT images. Other LNs (with a short diameter of 4-6 mm) lacked obvious metastatic signs on CT images, so the radiologist had difficulty in diagnosing them. Suspicious radiologic features of malignant LNs on CT images included cystic changes, calcifications, rounded shape, short diameter longer than $5 \mathrm{~mm}$, obvious enhancement, and nodular enhancement.

\section{Patbological examination}

Pathological diagnosis is the golden criterion for assessing the status of LNs. The patients in this study underwent central and/or lateral LN dissection. These level VI LNs were marked as the prelaryngeal, the pretracheal, the right and the left paratracheal nodes during operation separately, and then all resected cervical LNs were sent for pathological examination.

\section{Statistical analysis}

The SPSS software (version 22.0) was used for statistical analysis. The quantitative parameters between benign and malignant LNs were compared with Student's $t$-test. $\mathrm{P}<0.05$ was considered statistically significant for these parameters. The quantitative parameters were fitted to univariable and multivariable binary logistic regression models. Univariable binary logistic regression analysis was used to determine the ability of each parameter to differentiate between metastatic and benign LNs. Spectral parameters with a $\mathrm{P}$ value less than 0.05 in the univariate binary logistic regression analysis were included for multivariable logistic regression analysis. Multivariable binary logistic regression analysis was performed to determine the independent predictive parameters for the diagnosis of $\mathrm{LN}$ metastasis, and a forward step-wise selection was applied by using the likelihood-ratio test for variable selection.

The diagnostic role of quantitative parameters for small lymph nodes was analyzed using the receiver operating characteristic (ROC) curves and compared using the McNemar test. The diagnostic efficiency of the parameter model was calculated by using the area under the curve (AUC) values from ROC curves. The Calibration Plot was also performed to evaluate the parameter model by using Hosmer-Lemeshow good of fit test. The optimal threshold was determined by using the Youden index (sensitivity + specificity-1). The sensitivity, specificity, PPV, NPV, and accuracy of the quantitative dual-energy CT parameters were also calculated.

\section{Results}

Of all the 74 patients, 34 were women and 40 were men, with a mean age of 42.3 years (range, 13-67 years). LNs from the benign group were distributed diffusely in levels II, III, IV, and VI, whereas the LNs in the malignant group were in levels IV and VI. A total of 216 lymph nodes, including 92 (42.6\%) metastatic LNs from 43 patients with PTC and 124 (57.4\%) benign LNs from 31 patients with thyroid disease, were found on the preoperative CT images. LNs with a short diameter of 2-4 $\mathrm{mm}$ accounted for $75.92 \%$ of all LNs, and the average short diameter of these lymph nodes was $3.6 \mathrm{~mm}$. Thirteen benign lymph nodes were in level II and III, 9 LNs were in level IV, and 194 LNs were in level VI.

The diagnostic error rate and accuracy rate was $9.7 \%$ (21 LNs) and $90.3 \%$ for the parameter model based on dual-energy CT parameters, respectively. This model had false-positive results in $11 \mathrm{LNs}$ and false-negative results in $10 \mathrm{LNs}$. The diagnostic performances of the parameter model and image model are shown in Table 1.

Of the 113 small lymph nodes with a short diameter of $2-3 \mathrm{~mm}$, the radiologists believed that only 30 of them could be diagnosed using conventional CT images at small FOV, of which 25 LNs were correctly diagnosed and 5 were misdiagnosed; while the remaining 83 lymph nodes did not show visible signs of metastasis on CT images. Some 
Table 1 The diagnostic performance of quantitative dual-energy CT parameters model and CT images at small FOV

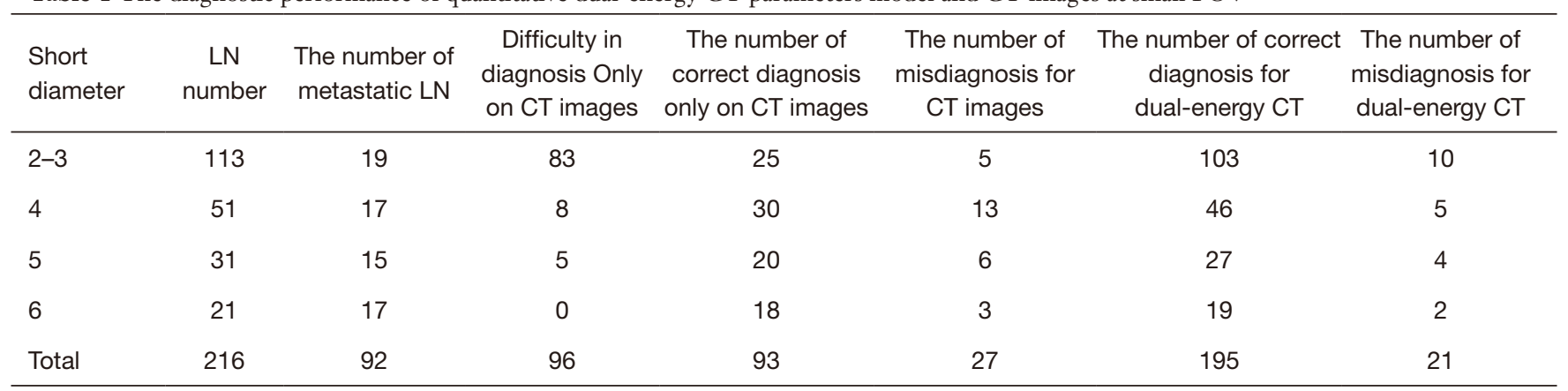

Among the lymph nodes diagnosed with dual-energy CT parameters model, the predicted diagnostic values of 4 false negative lymph nodes and 2 false positive lymph nodes were very close to the diagnostic threshold. CT, computed tomography.

Table 2 Diagnostic performance of multiple parameters model in diagnosing small lymph nodes

\begin{tabular}{lc}
\hline Parameters & $\begin{array}{c}\text { Diagnostic model of dual-energy CT } \\
\text { parameters }\end{array}$ \\
\hline No. of true positive & 77 \\
No. of false positive & 6 \\
No. of true negative & 118 \\
No. of false negative & 15 \\
Sensitivity (\%) & 83.7 \\
Specificity (\%) & 95.2 \\
Accuracy (\%) & 90.3 \\
PPV (\%) & 92.8 \\
NPV (\%) & 88.7 \\
\hline
\end{tabular}

of them were near the thyroid cancer in level VI, and their metastatic status could not be judged. Eight lymph nodes with a short-diameter of $4 \mathrm{~mm}$ and 5 lymph nodes with a short-diameter of $5 \mathrm{~mm}$ did not show any metastatic sign on CT images, making it difficult to diagnose the LN status. Among all these lymph nodes, the diagnostic accuracy of the image model was $43.1 \%$ and the diagnostic error rate was $12.5 \%$. However, the diagnostic difficulty rate was $44.4 \%$. In small lymph nodes with a short diameter of 2-3 $\mathrm{mm}$, the probability of accurate diagnosis was only $22.1 \%$ and the diagnosis difficulty rate was $73.5 \%$.

\section{Univariate analysis}

Table 2 shows the diagnostic value of the parameter model in diagnosing small lymph nodes after calculating the diagnostic value of each cut-off point for multiple parameters. Quantitative dual-energy CT parameters in discriminating between the benign lymph nodes and malignant lymph nodes are shown in Table 3. Representative contrast enhanced CT images showed that IC, spectral curve, and effective atomic number were different in benign and metastatic lymph nodes (Figure 2).

It is showed that the $\mathrm{P}$ value of multiple spectral parameters were $<0.05$ between benign and malignant LNs (Table 3), and they were included for the binary logistic regression analysis. Only four parameters showed no statistical difference. ROC analysis of quantitative dual-energy CT parameters for differential diagnosis of metastatic and non-metastatic lymph nodes and their diagnostic performances are shown in Table 4. The arterial phase IC had the highest diagnostic accuracy $(85.65 \%)$ on a per-LN basis. The arterial phase NIC had a higher AUC value than other parameters.

\section{Binary logistic regression model}

Several independent variables demonstrated statistical significance between benign and malignant LNs. According to the binary logistic regression analysis, we built the following model with dual-energy CT parameters for predicting metastatic lymph nodes using the following formula:

Probability of malignant $\mathrm{LNs}=\mathrm{e}^{\mathrm{y}} /\left(1+\mathrm{e}^{\mathrm{y}}\right)$, where $\mathrm{y}=$ $-23.119+0.033 \times$ precontrast phase Rho $+0.076 \times$ arterial phase NIC $+2.156 \times$ arterial phase $Z-0.540 \times$ venous phase $\mathrm{K}+1.676 \times$ venous phase IC.

We found that pre-contrast electron cloud density, normalized IC in the arterial phase, effective atomic number in the arterial phase, $\lambda_{\mathrm{HU}}$ in the venous phase, and IC in the 
Table 3 Quantitative dual-energy CT parameters in discriminating between metastatic and non-metastatic lymph nodes

\begin{tabular}{|c|c|c|c|}
\hline Parameter & Benign lymph nodes & Metastatic lymph nodes & $P$ value \\
\hline Precontrast IC & $0.7 \pm 0.3$ & $0.8 \pm 0.7$ & 0.434 \\
\hline Precontrast Rho & $29.1 \pm 8.3$ & $34.7 \pm 21.6$ & 0.019 \\
\hline Precontrast Z & $7.7 \pm 0.2$ & $7.9 \pm 0.4$ & 0.001 \\
\hline Arterial phase $\lambda_{\mathrm{HU}}$ & $2.3 \pm 1.0$ & $5.0 \pm 2.5$ & 0.000 \\
\hline Arterial phase IC (mg/mL) & $1.4 \pm 0.6$ & $3.1 \pm 1.4$ & 0.000 \\
\hline Arterial phase NIC (\%) & $16.8 \pm 8.5$ & $38.2 \pm 17.9$ & 0.000 \\
\hline Arterial phase Rho & $31.7 \pm 9.3$ & $35.5 \pm 23.3$ & 0.131 \\
\hline Venous phase $\lambda_{\mathrm{HU}}$ & $3.6 \pm 1.1$ & $5.3 \pm 1.8$ & 0.000 \\
\hline Venous phase IC (mg/mL) & $2.0 \pm 0.6$ & $3.1 \pm 1.0$ & 0.000 \\
\hline Venous phase NIC (\%) & $38.5 \pm 13.0$ & $61.4 \pm 23.8$ & 0.000 \\
\hline Venous phase Rho & $33.0 \pm 10.1$ & $36.2 \pm 19.0$ & 0.147 \\
\hline Venous phase $Z$ & $8.5 \pm 0.3$ & $9.0 \pm 0.4$ & 0.000 \\
\hline Venous phase DEI & $0.021 \pm 0.007$ & $0.030 \pm 0.009$ & 0.000 \\
\hline
\end{tabular}

Quantitative variables are in mean \pm SD. CT, computed tomography; $\lambda_{\mathrm{HU}}$, slope of the spectral Hounsfield unit curve; IC, iodine concentration; NIC, normalized iodine concentration; Z, effective atomic number; Rho, electron cloud density; DEI, double energy index.

venous phase had the best diagnostic value. The AUC was 92\% for the parameter model (Figure 3). Figure 4 shows the assessment of this parameter model, which indicated that the predicted value was close to the observed value in both the benign LN group and malignant LN group for this diagnostic model. Logistic regression analysis of metastatic status results is shown in Table 5 .

\section{Discussion}

There have been dual-energy CT studies investigating lymph node metastasis $(3,4)$, and several studies have found that CT has additional benefits for lymph node metastasis in thyroid cancer (5). However, there is still a diagnostic dilemma in the diagnosis of small LNs. The smaller the lymph nodes are, the more difficult it may become to diagnose. Especially when one lymph node is $<3 \mathrm{~mm}$ in short diameter, it becomes more difficult to determine the metastatic status of small LNs. About half of the 216 small lymph nodes were difficult to diagnose only on conventional
CT images at small FOV. While most of the 216 small LNs could be diagnosed correctly with our parameter model. We found that the parameter model had high accuracy and diagnostic values in diagnosing small lymph nodes of thyroid cancer, which was better than that of the image model based on conventional CT images at small FOV, especially for lymph nodes with short diameter $<3 \mathrm{~mm}$. Our diagnostic accuracy of LN status is also better than that in previous studies (5-9). We have used AUC and calibration curve to evaluate the discrimination and calibration of the parameter model, and its discrimination and calibration are excellent.

Compared with previous dual-energy CT studies, the dual-energy CT scanner we used belonged to the third generation, which improved the energy resolution and the ability to distinguish substances; and we also investigated some new parameters, including electron cloud density, effective atomic number, and DEI in three phases, to develop a parameter model. We performed dual-source dual-energy CT at small FOV with multiple quantitative 

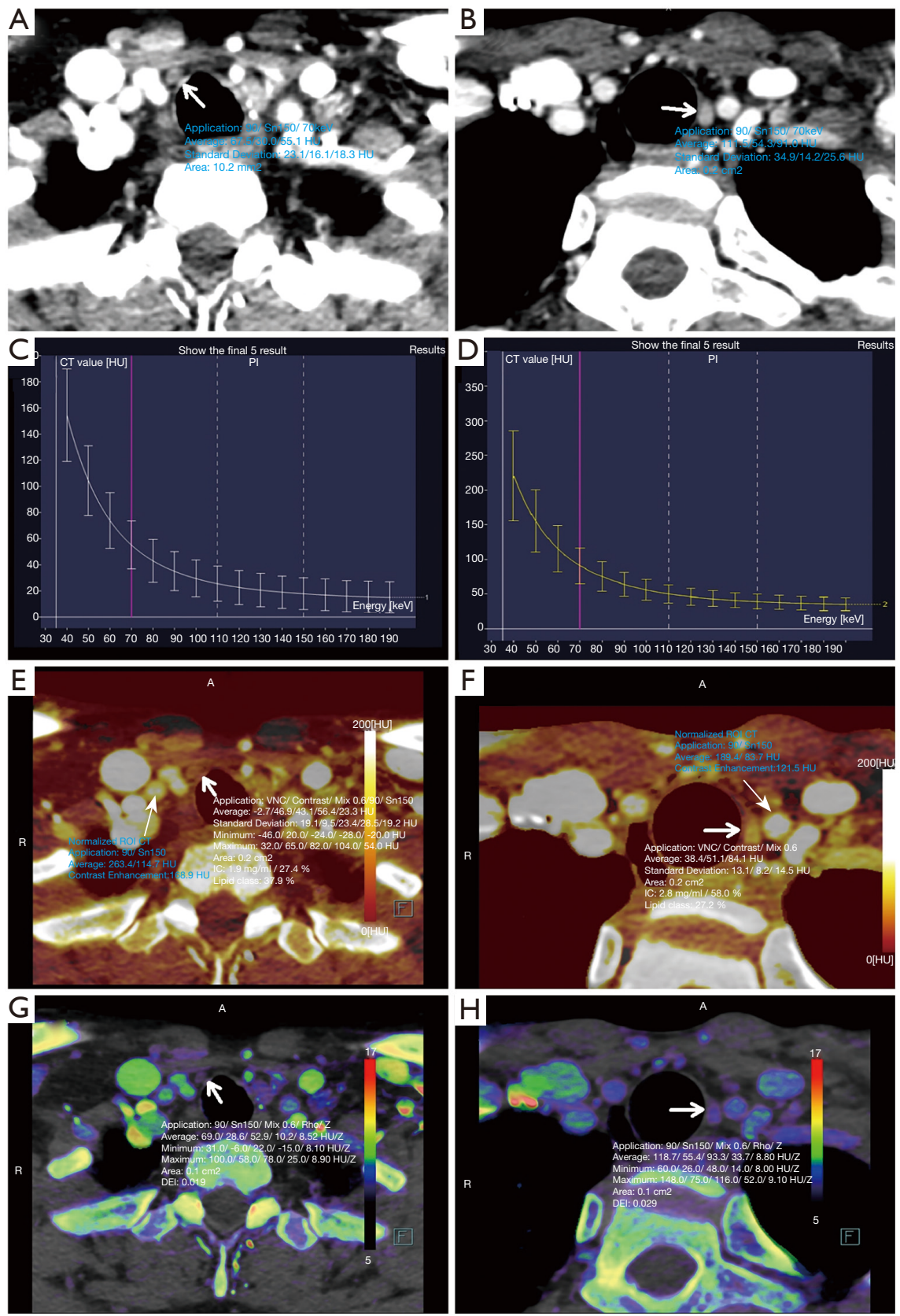

Figure 2 Representative CT images at small FOV and measurement of dual-energy CT parameters in two different patients for small lymph nodes. Images of benign lymph nodes were in the left column, and images of malignant lymph node were in the right column. Representative venous phase contrast-enhanced dual-energy CT images show the target lymph node and $\lambda_{\mathrm{HU}}$ in non-metastatic and metastatic lymph nodes (A-D). (E-H) Representative iodine concentration images and effective atomic number images at small FOV (or effective atomic number images), which outlines the target lymph node and measures iodine concentration and effective atomic number. (A) representative venous phase CT images of a benign lymph node. (B) Representative venous phase CT images of a malignant lymph node. (C) Graph shows spectral HU curves of benign lymph node. (D) Graph shows spectral HU curves of malignant lymph node. (E) Iodine-based pseudo-colorized image of a benign lymph node; (F) Iodine-based pseudo-colorized image of a metastatic lymph node. (G) Representative effective atomic number images of a benign lymph node. $(\mathrm{H})$ Representative effective atomic number images of a metastatic lymph node. CT, computed tomography; HU, hounsfield unit. 
Table 4 The prediction of LN status results and performance comparison of different quantitative dual-energy CT parameters

\begin{tabular}{|c|c|c|c|c|c|c|c|}
\hline Parameters & AUC & Threshold & Sensitivity (\%) & Specificity (\%) & PPV (\%) & NPV (\%) & Accuracy (\%) \\
\hline Precontrast Rho & $0.633(0.553-0.714)$ & 34.85 & 55.4 & 73.4 & 60.7 & 68.9 & 65.74 \\
\hline Precontrast Z & $0.669(0.589-0.749)$ & 7.955 & 42.4 & 96.8 & 90.7 & 69.4 & 73.61 \\
\hline Precontrast DEI & $0.688(0.609-0.767)$ & 0.0095 & 50.0 & 88.7 & 76.7 & 70.5 & 72.22 \\
\hline Arterial phase IC (mg/mL) & $0.897(0.849-0.944)$ & 1.95 & 84.8 & 86.3 & 82.1 & 88.4 & 85.65 \\
\hline Arterial phase NIC (\%) & $0.899(0.853-0.945)$ & 22.75 & 82.6 & 86.3 & 81.7 & 87.0 & 84.72 \\
\hline Arterial phase Rho & $0.610(0.528-0.692)$ & 42.9 & 38.0 & 92.7 & 79.5 & 66.9 & 69.44 \\
\hline Arterial phase Z & $0.881(0.831-0.932)$ & 8.335 & 83.7 & 81.5 & 77 & 87.1 & 82.41 \\
\hline Venous phase IC (mg/mL) & $0.854(0.801-0.908)$ & 2.45 & 80.4 & 78.2 & 73.3 & 84.3 & 79.17 \\
\hline Venous phase NIC (\%) & $0.819(0.760-0.879)$ & 44.7 & 80.4 & 70.2 & 66.7 & 82.9 & 74.54 \\
\hline Venous phase Rho & $0.606(0.525-0.687)$ & 44.4 & 39.1 & 90.3 & 75 & 66.7 & 68.52 \\
\hline Venous phase Z & $0.818(0.757-0.878)$ & 8.74 & 75.0 & 78.2 & 71.9 & 80.8 & 76.85 \\
\hline Venous phase DEI & $0.790(0.727-0.853)$ & 0.0235 & 82.6 & 65.3 & 63.9 & 83.5 & 72.69 \\
\hline
\end{tabular}

Data in parentheses are $95 \% \mathrm{Cls}$ of AUC. AUC, area under the receiver operating characteristic curve; CT, computed tomography; PPV, positive predictive value; NPV, negative predictive value; $\lambda_{H U}$, slope of the spectral Hounsfield unit curve; IC, iodine concentration; NIC, normalized iodine concentration; Z, effective atomic number; Rho, electron cloud density; DEl, double energy index.



Figure 3 ROC curve of quantitative dual-energy CT parameters model. Best threshold value for predictive probability of binary logistic regression model of multiple parameters in differentiating benign from metastatic lymph nodes was 0.4897457 . AUC = area under the curve. AUC $=0.924$ (95\% CI: 0.881-0.967). The parameter model showed good discrimination. CT, computed tomography.

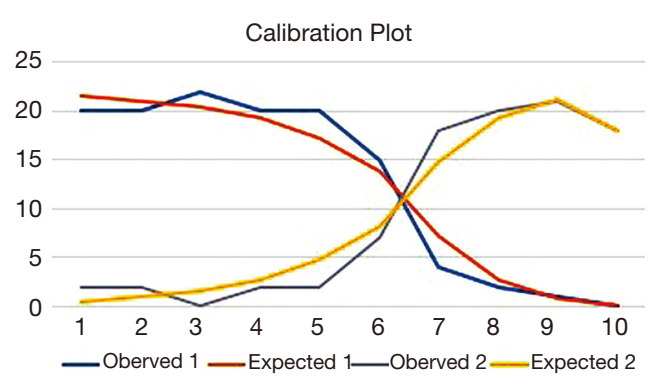

Figure 4 The calibration for dual-energy parameters model. The diagnostic model indicated that the predicted value is close to the observed value in the benign LN group and malignant LN group. Observed value 1 and expected value 1 belongs to benign lymph node; Observed value 2 and expected value 2 belongs to the malignant lymph node. The results of Hosmer-Lemeshow good of fit test were as following: Chi-Square was 12.811 , with $\mathrm{P}=0.119$.

parameters to facilitate accurate detection and diagnosis of small lymph nodes. This study uses thin-section images at small FOV with improved resolution, and it helps identify small lymph nodes and the delineation of conformal ROI. 
Table 5 Logistic regression analysis of metastasis status results

\begin{tabular}{|c|c|c|c|c|c|c|c|c|}
\hline Independent variable & $\mathrm{B}$ & S.E. & Wals & $\mathrm{df}$ & Sig. & $\operatorname{Exp}(B)$ & \multicolumn{2}{|c|}{$\operatorname{EXP}(\mathrm{B}) 95 \% \mathrm{Cl}$} \\
\hline Precontrast Rho & 0.033 & 0.017 & 3.477 & 1 & 0.062 & 1.033 & 0.998 & 1.069 \\
\hline Arterial phase NIC & 0.076 & 0.022 & 12.568 & 1 & 0.000 & 1.079 & 1.035 & 1.126 \\
\hline Arterial phase Z & 2.156 & 0.643 & 11.241 & 1 & 0.001 & 8.641 & 2.449 & 30.482 \\
\hline Venous phase $\mathrm{IC}(\mathrm{mg} / \mathrm{mL})$ & 1.676 & 0.550 & 9.274 & 1 & 0.002 & 5.344 & 1.817 & 15.716 \\
\hline Constant & -23.119 & 5.167 & 20.020 & 1 & 0.000 & 0.000 & & \\
\hline
\end{tabular}

B, beta ( $\beta$ ) coefficient; S.E., standard error; Wals, Wald chi-square; df, degree of freedom; Sig., significance ( $P$ value); Exp(B), odds ratio; $\mathrm{Cl}$, confidence interval; $\lambda_{\mathrm{HU}}$, slope of the spectral Hounsfield unit curve; IC, iodine concentration; NIC, normalized iodine concentration; Z, effective atomic number; Rho, electron cloud density.

Its boundary can be identified more accurately, which is beneficial to multi-parameter measurement and diagnosis of small lymph nodes with dual-energy CT. Small FOV, thinsection, and multi-planar reconstruction can help observe cervical LNs from different planes, detect more small LNs and discover more features in one LN, which help to diagnose metastatic LN (8).

CARE Dose 4D or iterative reconstruction algorithm can reduce the radiation dose so that the radiation dose of CT at small FOV is not higher than that of $\mathrm{CT}$ at conventional FOV; dual-source dual-energy CT with energy spectrum purification technique reduces the radiation dose $(10,11)$, leading to a $30 \%$ radiation dose reduction.

Macrometastatic LNs must be treated, because it leads to a worse prognosis, while benign cervical lymph nodes of thyroid cancer do not require surgical resection. Some scholars believed that after the removal of micrometastatic lymph nodes (2), the prognosis was similar to that of benign LNs (1). For patients with thyroid cancer and micrometastatic lymph nodes, if these LNs are not resected, is the prognosis really the same as that of thyroid cancer patients without LN metastasis? No studies have ever provided the answer. And micrometastatic LNs may develop into macrometastatic LNs or metastasis in other sites. So, the diagnosis of micrometastatic LNs and very small LN metastasis should be investigated, and its management may be different from that of benign or macrometastatic LNs. Should active surveillance be adopted for micrometastatic LNs? The methods we used in our study may be helpful and inspiring for the diagnosis of small LN or micrometastatic LN.

Central compartment lymph nodes are believed to be the first stage of lymph node metastasis in thyroid cancer $(6,12)$. Most lymph nodes were located in level VI in our study, and small lymph nodes account for the majority of them. For small FOV CT images, the signal-to-noise ratio and spatial resolution have improved (8,13-16).

ROI was often drawn in the enhancement area in previous studies, and this area did not necessarily represent the entire lymph node. We usually encompass the whole lymph node as ROI, not just a small part of it. Our ROI delineation of lymph nodes was different from that of previous studies. When we measured and calculated the slope of the spectral Hounsfield unit curve, our ROI at least included most part of the LN; while measuring other quantitative parameters of dual-energy CT, we tried to make the ROI include the entire lymph node, rather than delineating the typical part or the central part of one lymph node. And we believed that this method of ROI delineation was more comprehensive, accurate, and representative. In many studies $(3,4,17)$, the ROI of lymph node excluded cystic, necrotic, and calcification areas, while our delineation included all areas in the target lymph node. We believe that in most thyroid cancer cases, the calcification and cystic changes in one lymph node represent the characteristics of metastatic foci, and these typical areas should be included in the ROI, in case of the spectral parameters can fully reflect the true characteristics of lymph node metastasis in thyroid cancer. Further, our delineation area included the marginal part of the lymph node, and the marginal part of the lymph node was often the first or most common site for tumor deposits. Pathological examination showed that about $57 \%$ of tumor deposits in $\mathrm{LN}$ were located at the marginal sinus (18), which was usually at the marginal part of one 
lymph node. Our ROI, including the commonest site of tumor deposits in lymph node may better reflect whether the lymph node is metastatic.

The method we used for LN selection made the pathological results of the chosen $\mathrm{LN}$ reliable, and we could precisely compare the predicted results of multiple parameters model with pathological results to investigate the diagnostic value of dual-energy CT at small FOV. Because the pathological results of all LNs at the same level or sublevel were the same, we could select any of these small lymph nodes for multiple quantitative parameters measurement to ensure the radiologic-pathologic correlation reliable. The method reduced the tedious procedures of the one node-by-one node correlation between CT and pathology, and it lessened the probability of possible errors (4), improving the quality of the study. All selected lymph nodes had definite pathological results in our research. The thyroid surgeon in our team routinely divided bilateral cervical level VI further into four sublevels for patients with thyroid disease preoperatively: pre-laryngeal, pre-tracheal, left paratracheal, and right paratracheal sublevels (19). And then, these cervical nodes were resected and labeled; we measured multiple quantitative parameters of dual-energy CT and performed a diagnostic model. More sublevels with LNs could be screened and enrolled in this study.

This is a retrospective diagnostic study, and there may be selection bias. Prospective research is required to further validate the parameter model, and we have registered a prospective study (Registration number: ChiCTR2000035195; http://www.chictr.org.cn). Random selection of targeted lymph nodes for one-by-one correspondence between imaging and pathology can help eliminate selection bias.

\section{Conclusions}

We believe that by using dual-source dual-energy CT at small FOV, the parameter model can accurately predict the metastasis of small lymph node in thyroid cancer, which is superior to the image model and previous studies, and it has increased diagnostic accuracy. Quantitative dual-energy CT parameters are very useful for small lymph nodes that were difficult to be diagnosed only on conventional CT images. The parameter model has better discrimination and allows more small LNs to be identified. Accurate assessment of cervical LN status does good to cancer staging and treatment.

\section{Acknowledgments}

We would like to thank Xiao Linling and Seeruttun Sharvesh Raj for their help in language editing. Funding: None.

\section{Footnote}

Reporting Checklist: The authors have completed the STARD reporting checklist. Available at http://dx.doi.org/10.21037/ gs-20-822

Data Sharing Statement: Available at http://dx.doi. org/10.21037/gs-20-822

Conflicts of Interest: All authors have completed the ICMJE uniform disclosure form (available at http://dx.doi. org/10.21037/gs-20-822). The authors have no conflicts of interest to declare.

Ethical Statement: The authors are accountable for all aspects of the work in ensuring that questions related to the accuracy or integrity of any part of the work are appropriately investigated and resolved. The study was conducted in accordance with the Declaration of Helsinki (as revised in 2013). This was a single-center retrospective diagnostic study, approved by the institutional ethics committee of Sun Yat-sen University Cancer Center (IRB number: B2020-156), and informed consent was waived.

Open Access Statement: This is an Open Access article distributed in accordance with the Creative Commons Attribution-NonCommercial-NoDerivs 4.0 International License (CC BY-NC-ND 4.0), which permits the noncommercial replication and distribution of the article with the strict proviso that no changes or edits are made and the original work is properly cited (including links to both the formal publication through the relevant DOI and the license). See: https://creativecommons.org/licenses/by-nc-nd/4.0/.

\section{References}

1. Haugen BR, Alexander EK, Bible KC, et al. 2015 American Thyroid Association Management Guidelines for Adult Patients with Thyroid Nodules and Differentiated Thyroid Cancer: The American Thyroid Association Guidelines Task Force on Thyroid Nodules and Differentiated Thyroid Cancer. Thyroid 2016;26:1-133. 
2. Amin MB, Edge S, Greene F, et al. AJCC Cancer Staging Manual. 8 ed. New York: Springer International Publishing, 2017.

3. Liu X, Ouyang D, Li H, et al. Papillary thyroid cancer: dual-energy spectral CT quantitative parameters for preoperative diagnosis of metastasis to the cervical lymph nodes. Radiology 2015;275:167-76.

4. Zhang X, Zheng C, Yang Z, et al. Axillary Sentinel Lymph Nodes in Breast Cancer: Quantitative Evaluation at DualEnergy CT. Radiology 2018;289:337-46.

5. Lee DH, Kim YK, Yu HW, et al. Computed Tomography for Detecting Cervical Lymph Node Metastasis in Patients Who Have Papillary Thyroid Microcarcinoma with Tumor Characteristics Appropriate for Active Surveillance. Thyroid 2019;29:1653-9.

6. Lesnik D, Cunnane ME, Zurakowski D, et al. Papillary thyroid carcinoma nodal surgery directed by a preoperative radiographic map utilizing CT scan and ultrasound in all primary and reoperative patients. Head Neck 2014;36:191-202.

7. Jeong HS, Baek CH, Son YI, et al. Integrated 18F-FDG PET/CT for the initial evaluation of cervical node level of patients with papillary thyroid carcinoma: comparison with ultrasound and contrast-enhanced CT. Clin Endocrinol (Oxf) 2006;65:402-7.

8. Cho SJ, Suh CH, Baek JH, et al. Diagnostic performance of CT in detection of metastatic cervical lymph nodes in patients with thyroid cancer: a systematic review and metaanalysis. Eur Radiol 2019;29:4635-47.

9. Zhou Y, Su GY, Hu H, et al. Radiomics analysis of dualenergy CT-derived iodine maps for diagnosing metastatic cervical lymph nodes in patients with papillary thyroid cancer. Eur Radiol 2020;30:6251-62.

10. Pulickal GG, Singh D, Lohan R, et al. Dual-Source Dual-Energy CT in Submandibular Sialolithiasis: Reliability and Radiation Burden. AJR Am J Roentgenol 2019;213:1291-6.

Cite this article as: Zhuo S, Sun J, Chang J, Liu L, Li S. Dualsource dual-energy thin-section CT combined with small field of view technique for small lymph node in thyroid cancer: a retrospective diagnostic study. Gland Surg 2021;10(4):1347-1358. doi: $10.21037 / g s-20-822$
11. Tabari A, Gee MS, Singh R, et al. Reducing Radiation Dose and Contrast Medium Volume With Application of Dual-Energy CT in Children and Young Adults. AJR Am J Roentgenol 2020;214:1199-205.

12. Zhao H, Li H. Meta-analysis of ultrasound for cervical lymph nodes in papillary thyroid cancer: Diagnosis of central and lateral compartment nodal metastases. Eur J Radiol 2019;112:14-21.

13. Miyata T, Yanagawa M, Hata A, et al. Influence of field of view size on image quality: ultra-high-resolution CT vs. conventional high-resolution CT. Eur Radiol 2020;30:3324-33.

14. Sheshadri A, Rodriguez A, Chen R, et al. Effect of Reducing Field of View on Multidetector Quantitative Computed Tomography Parameters of Airway Wall Thickness in Asthma. J Comput Assist Tomogr 2015;39:584-90.

15. Zhu H, Zhang L, Wang Y, et al. Improved image quality and diagnostic potential using ultra-high-resolution computed tomography of the lung with small scan FOV: A prospective study. PLoS One 2017;12:e0172688.

16. Bechara B, McMahan CA, Moore WS, et al. Contrast-tonoise ratio with different large volumes in a cone-beam computerized tomography machine: an in vitro study. Oral Surg Oral Med Oral Pathol Oral Radiol 2012;114:658-65.

17. Li L, Cheng SN, Zhao YF, et al. Diagnostic accuracy of single-source dual-energy computed tomography and ultrasonography for detection of lateral cervical lymph node metastases of papillary thyroid carcinoma. J Thorac Dis 2019;11:5032-41.

18. Yanagita S, Natsugoe S, Uenosono Y, et al. Morphological distribution of metastatic foci in sentinel lymph nodes with gastric cancer. Ann Surg Oncol 2008;15:770-6.

19. Liu T, Su X, Chen W, et al. A mathematical model using computed tomography for the diagnosis of metastatic central compartment lymph nodes in papillary thyroid carcinoma. Eur Radiol 2014;24:2827-34. 